Session 12-1

\title{
Smart Design of IR Scene Generation Using Metallic Photonic Crystals
}

\author{
Gonzalo B. Farfan \\ Electrical \& Computer Engineering Department \\ University of New Mexico \\ Roshan R. Rammohan \\ Computer Science Department \\ University of New Mexico \\ Mahmoud R. Taha \\ Civil Engineering Department \\ University of New Mexico \\ Ihab El-Kady \\ Photonics Microsystems Technologies Department \\ Sandia National Laboratories
}

\begin{abstract}
Photonic Crystals (PCs) are nano-structures with a unique ability of controlling light propagation. One of the most difficult objectives to achieve in the design of this material is a desired performance of the light spectrum over the PCs. The geometry of the PC and its dimensions are variables that influence the spectral behavior of this crystal. Analytical and numerical methods exist for simulating spectral response of the PC for a given crystal geometry. However, the reverse problem is analytically intractable. In this work we propose a unique hybrid-optimization technique, based on Genetic Algorithms (GA) and Gradient Descent (GD) methods, for the smart design of photonic crystal emitters. We describe the photonic simulation method, and consider the granularity of the photonic crystal dimensions that control the design of the emitter. We demonstrate an innovative sliding-window method for performing a local heuristic search, and show how the proposed method improves the convergence rate of the genetic optimization process. We also show that with this technique caching spectral simulations is possible, and demonstrate how the caching becomes indispensable in an optimization process that has a computationally-expensive objective evaluation routine. Finally, we demonstrate the application of the proposed method on a case study for the design of a multi-band photonic emitter made of four independent tungsten-Lincoln-log photonic crystals. With the implementation of proposed optimization methods, an average emitter efficiency of $50 \%$ is achieved over a wide range of infrared wavelengths. We especially note the ability of the optimal photonic crystal structure, designed using the proposed method, to produce an unprecedented high emission efficiency of $54.5 \%$ in a significantly long wavelength region. Such a high efficiency at this wavelength has not been reported before in the literature.
\end{abstract}




\section{Introduction}

Photonic Crystals (PCs) are a new and promising technology that could enable high efficiency emission devices and fast computing devices. Composed of periodic dielectric or metallic-cumdielectric structures as shown in Fig. 1, PCs are designed to control the propagation of electromagnetic waves (EM) in ways similar to how the periodic potential in a semiconductor crystal controls electron motion by defining allowed and forbidden electronic energy bands. An electromagnetic wave passing throughout an array of periodic scatterers will undergo destructive interference for certain combinations of wave-vectors at certain frequencies, thus forbidding propagation for such wave-vectors at these frequencies ${ }^{1,2}$. To employ PC as independent IR emitters with pre-specified bands. Namely we want to limit emission in distinct band widths. The geometry of the PC and its dimensions are variables that influence the spectral behavior of this crystal. Analytical and numerical methods exist for simulating spectral response of the PC for a given crystal geometry. However, the reverse problem is analytically intractable.

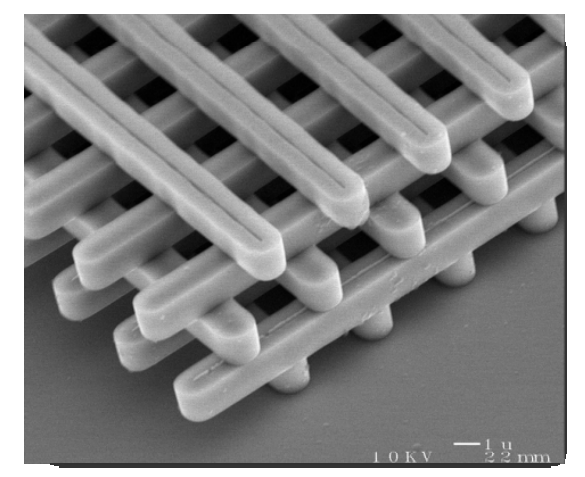

Fig. 1 SEM image of a Lincoln-log PC

\section{Background}

The spectral responses for a given photonic lattice are computed using the Rigorous Coupled Wave Analysis (RCWA) method. This can be performed by considering Maxwell's equations in the Fourier domain ${ }^{3}$.

$$
\begin{aligned}
& j \vec{k} \times \vec{E}=j \omega \mu \vec{H} \\
& j \vec{k} \times \vec{H}=-j \omega \varepsilon \vec{E}
\end{aligned}
$$

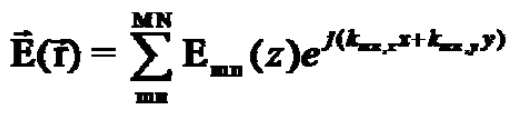

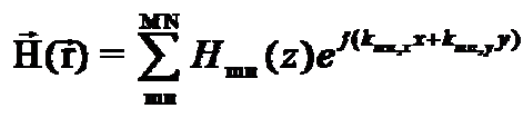

$$
\begin{aligned}
& \varepsilon(\vec{r})=\sum_{\mathrm{mz}}^{\mathrm{NN}} \varepsilon_{\mathrm{mm}}(z) e^{j \bar{\epsilon}_{\mathrm{mm}}-\vec{r}}
\end{aligned}
$$

RCWA expands $\mathrm{E}$ (electric field) and $\mathrm{H}$ (magnetic field) and $\varepsilon, \mu$ (parameters of electromagnetic properties) in series expansions. Using these fields a scattering matrix is computed. 
Each structure includes an 8 layer PC having a "Lincoln-log" geometry. These structures are assumed to be geometrically independent. As shown in Fig 2, our design variables are Lattice Constant (a), Rod Width (w) and Rod Thickness (h) ${ }^{4,5}$.

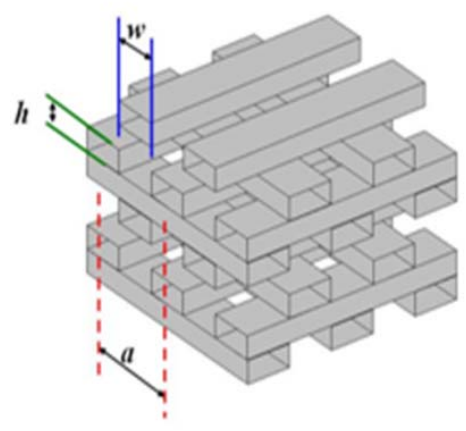

Fig. 2. A schematic of a Lincoln-log PC.

\section{Methods}

We solve this problem using a semi-natural Genetic Algorithm (GA), employing several heuristics to guide the search, caching prior attempts and interfering in the mutation process during evolution. The optimization process using these mathematical tools starts by establishing objective functions.To maximize the absorption and transmission profiles within a specific wavelength band and minimize it elsewhere, we established two objective functions:

1. RMS error between the desired and obtained responses (minimization)

2. Emitter efficiency with respect to black body radiation (maximization)

Our investigations showed that the emitter efficiency objective function can provide more sensitive and less noisy solutions compared with the RMS function.

Gradient descent optimization typically has slow performance and converges on local optima ${ }^{6}$. However, it helps eliminate some candidates and suggests a new starting population for the GA. Hence, our GA is "guided" by Gradient descent method. Genetic algorithms (GA) are known to be time consuming and resembling natural revolution by relying on mutations and crossovers between candidate solutions. Crossovers seem justifiable, however, our investigations showed a need to control and divert random mutation in search for smart solutions. We restrict the granularity of mutations to be consistent with real world fabrication constraints. We also make use of the prior knowledge that is based on physical principles of photonic crystals. For example, we replace some of the random mutations with an educated guess based on the sliding window scheme. (Fig 3a). 


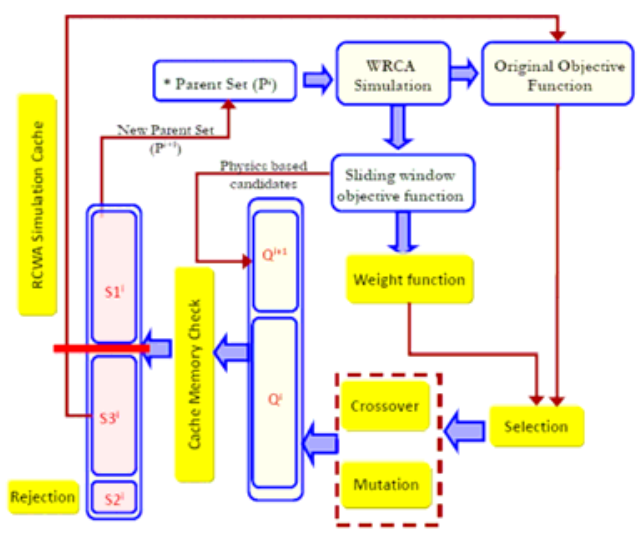

a)

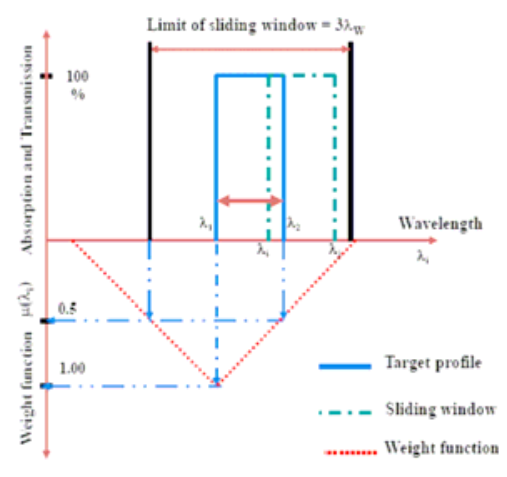

b)

Fig. 3 a) Semi-natural Genetic Algorithm b) Sliding window scheme

We therefore implement a spectral caching technique. While this process is less useful when searching in a bandgap region never searched before, it becomes increasingly useful in successive searches in the neighborhood, making the need for new simulation rare. A considerable reduction in computational time for optimization can be observed $(70 \%$ of total computational time) after a few instances of genetic optimization in a specific neighborhood. Fig. 4 shows how the spectral caching and the heuristic search methods are used to interfere in the evolution process. 


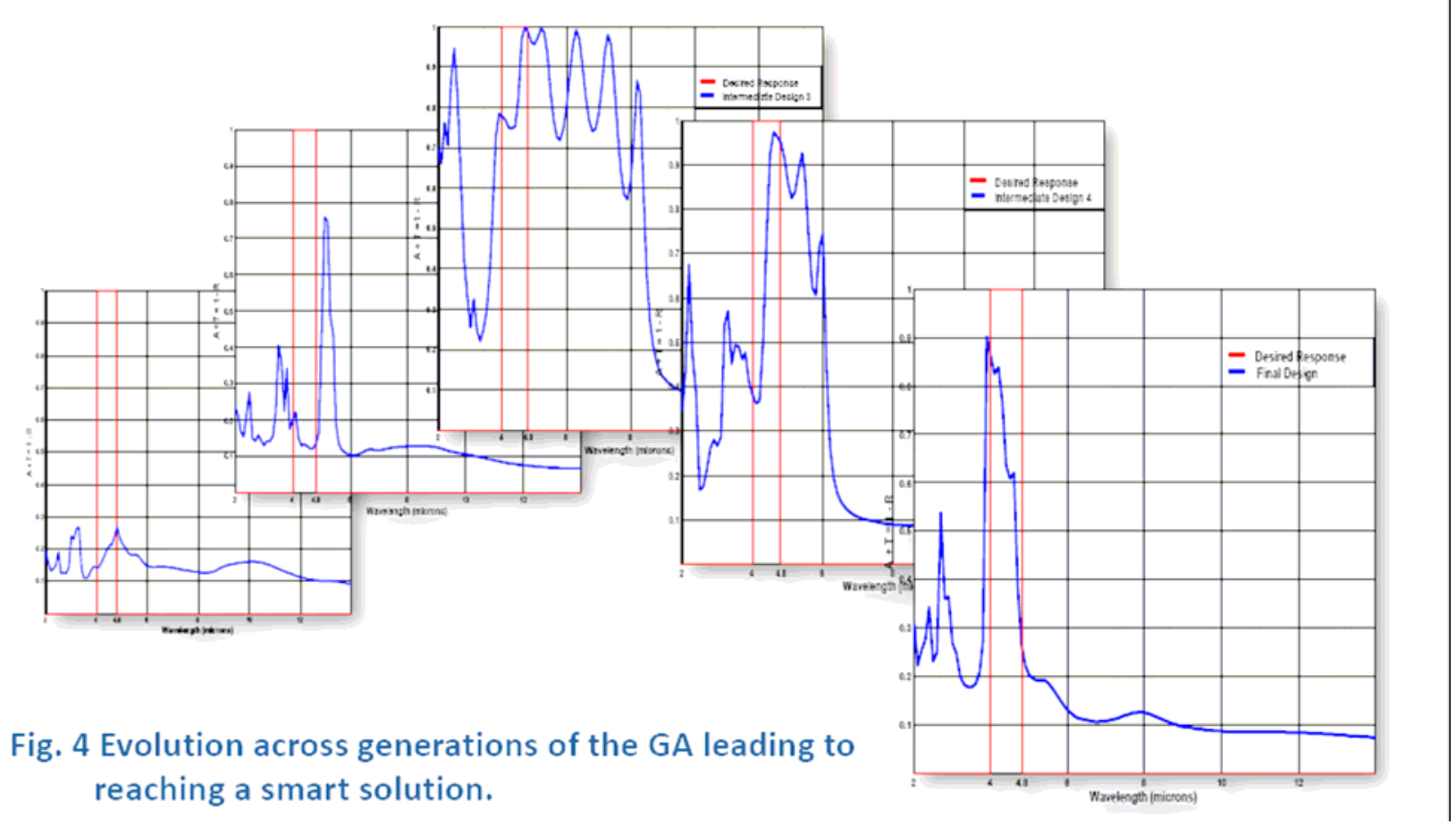

\section{Results and Conclusion}

The case of study is based on the analysis of three different desired bands. The results obtained after optimization are presented in Fig 5.The design that produced the responses in Fig 5 showed up at the genetic optimization stage, which underwent a modification of design by the sliding window local search heuristic to yield the response. In this section we report the quality of the design solutions achieved for the four bands in the multi-pixel photonic system described in the previous section.

Band 1 is intended to operate in the (3.45 - $4.45 \mathrm{um})$ wavelength range. The final design achieves an emitter efficiency of 53.9\% at 712K. With Band 2 (4 - $4.8 \mathrm{um}$ ), gradient descent proved more successful than with Pixel 1 and was able to provide a good starting point for the semi-natural genetic optimization. The final solution achieves an emitter efficiency of $38.9 \%$ at $694 \mathrm{~K}$ for the desired wavelength region. Band $3(8.5-9.6 \mathrm{um})$, the gradient descent provided a solution, as shown in Fig 5, that seemed little be towards the higher wavelength region, it was also observed to have a lot of high emission in the undesired lower wavelength regions. The semi-natural genetic optimization process provided the successive solutions. Finally Band 4 represents the most challenging design among the four pixels due to the well- known low emissivity of Tungsten for long wavelengths $(10-11.5 \mathrm{um})$. The application of the local search heuristic 
approach using the sliding window technique to replace some of the "natural" mutations, a surprisingly high emitter efficiency of $54.5 \%$ can be achieved.

The ability of a photonic lattice to manipulate the density of photon states and reduce the group velocity enabled the design of efficient IR emitters at wavelengths where small inherent material absorption would have prohibited it

Вand \&

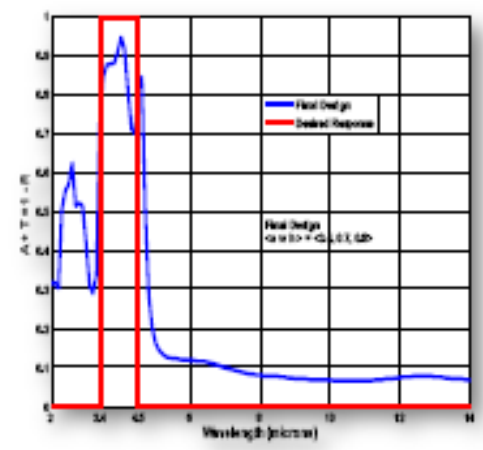

Bละด 3

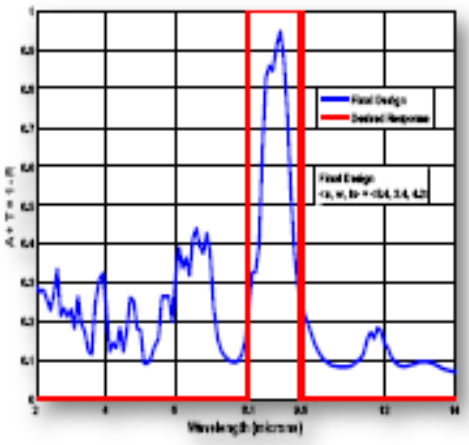

Band 2

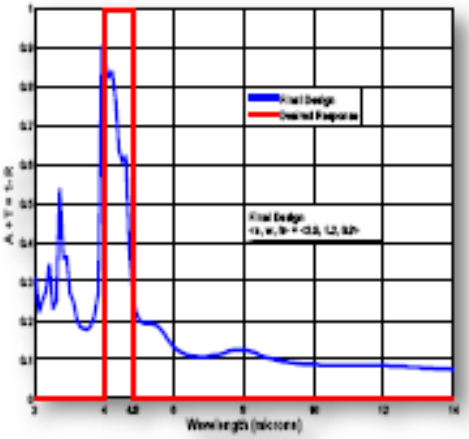

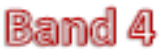

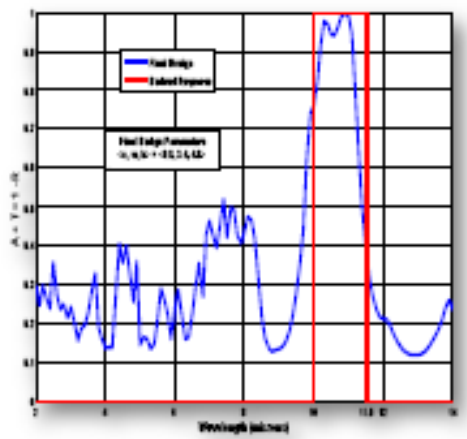

Fig. 5 Final design for each of the cases, showing the ability of the proposed method to achieve the pre-specified spectra for each accurately

\section{Future Work}

Further research is underway to develop thickness-restricted, dependent cases through the seminatural optimization method. To minimize angle spread, while still maintaining a network topology for energy recycling, we are investigating structures belonging to the face centered cubic (FCC) family. To reduce polarization dependence, we will investigate hexagonal based surfaces that possess a $\mathrm{C}_{6}$ axis. 


\section{Acknowledgements}

This work is supported by Sandia National Laboratories,(SNL). We greatly appreciate this support. Sandia National Laboratories (SNL), a multiprogram laboratory operated by Sandia Corporation, a Lockheed Martin Company, for the U.S. Department of Energy's National Nuclear Security Administration under contract DE-AC04-94AL85000. Use of the Center for Nanoscale Materials was supported by the U.S. Department of Energy, Office of Science, Office of Basic Energy Sciences, under Contract No. W-31-109-Eng-38.

\section{References}

1. Joannopoulos JD, Meade RD, Winn JN, Photonic Crystals: Molding the Flow of Light. ISBN13: 978-0-69103744-8. Princeton Univ. Press, 1995.

2. Johnson SG, and Joannopoulos, JD. Photonic Crystals - The Road from Theory to Practice. ISBN-13: 978-0792-37609-5. Kluwer, 2002.

3. Glytsis EN and Gaylord TK, Rigorous three-dimensional coupled-wave diffraction analysis of single and cascaded anisotropic gratings. J. Opt. Soc. Amer. A. Nov. 1987; vol. 4, pp. 2061-2080.

4. Yablonovitch E. Inhibited Spontaneous Emission in Solid-State Physics and Electronics. Physical Review Letters 1987; Vol. 58, 2059

5. Lin SY, Fleming JG and El-Kady I, Highly efficient light emission at $\mathrm{m} 5.1 \mu \lambda=$ by a three-dimensional tungsten photonic crystal. Optics Letters 2003; 28(18), 1683-1685.

6. Venkataraman, P., Applied Optimization with MATLAB Programming. John Wiley and Sons Inc. NY, USA, 2002 .

\section{GONZALO B. FARFAN}

Gonzalo B. Farfan received his B.S. degree in Electrical Engineering from the "Military School of Engineering" (La Paz-Bolivia) in 2005 and is continuing his studies as a M.S. student in the Electrical and Computer Engineering department at the University of New Mexico. His research includes optimization on nanostructure design.

\section{ROSHAN R. RAMMOHAN}

Roshan R. Rammohan received his B.E. degree in Instrumentation and Electronics Engineering from Bangalore University, India in 1999. After working in a Computational Neuroscience Lab in Bangalore, he moved to the University of New Mexico in 2002 where he is pursuing his Ph.D. in Computer Science. His research includes Artificial Intelligence, Machine Learning, Optimization Theory and Algorithms.

\section{MAHMOUD R. TAHA}

Mahmoud Reda Taha, is an Assistant Professor and Regents' Lecturer, Department of Civil Engineering, University of New Mexico. He received his BSC and MSC in structural engineering, from Ain Shams University, Cairo, Egypt in 1993 and 1996 respectively and his $\mathrm{PhD}$ from the University of Calgary in Canada in 2000. His research interests include structural health monitoring, nanotechnology for structural materials, modeling and simulation using artificial intelligence, and biomechanics. Dr. Taha was named as UNM Regents' Lecturer in 2007, the highest recognition to be bestowed on junior faculty members at UNM.

IHAB EL KADY

Ihab El-Kady is with the Department of Photonic Microsystems Technologies, Sandia National Laboratories in Albuquerque, NM, USA. 